\title{
Nasal Pain as an Aura to a Complex Partial Seizure
}

\author{
Egidio Spinelli, Abdullah Alkutbi, D. Barry Sinclair, Helly Goez
}

Keywords: Epilepsy - pediatric, pain, pediatric neurology

doi:10.1017/cjn.2015.357

Can J Neurol Sci. 2016; 43: 424-426

Pain as a primary or associated manifestation of seizures is a rare phenomenon. Reported as early as 1901 by Gowers, it has been divided into unilateral, cephalgic, and abdominal presentation and is thought to account for approximately 2.8 to 4.1 of seizures in pediatric and adult series. ${ }^{1,2}$ When unilateral, localization of painful seizures is mostly to the contralateral frontal, parietal, peri-Sylvian, and temporal areas. ${ }^{2}$ Abdominal pain is most consistently associated with temporal lobe epilepsy, whereas cephalgic pain localizes poorly. ${ }^{1}$ Pain can be a sole manifestation of a focal seizure or can be associated with alterations in consciousness and can evolve to bilateral convulsive seizures. When established, the etiology for these paroxysms tends to be structural with resolution of symptoms after surgical resection of the epileptogenic zone. Vasomotor changes with painful seizures are rare.

\section{Case History}

A 6-year-old right hand-dominant male presented to the emergency room with a 3-month history of episodes described as starting with a stinging sensation at the tip of the nose. The pain was followed by 30 to 40 seconds of facial flushing then pallor, with the entire episode lasting 40 to 50 seconds. Initially, the patient had an average of eight daily events mostly occurring during the daytime, which increased to more than 20 per day occurring both day and night. The pain would wake the patient during sleep and he had developed epistaxis of both nares as a result of rubbing both sides of his nose with either hand in pain.

Birth history was unremarkable. Medical history was significant for cryptogenic infantile spasms of unknown etiology diagnosed at 10 weeks of age and treated with vigabatrin. Spasms resolved at 2 years of age. Interim development was reported as normal by parents, although formal neuropsychiatric assessment was not done. Magnetic resonance imaging (MRI) of the brain at 3 months of age was normal. Examination revealed an alert and cooperative male with no dysmorphic features. Neurological examination was normal with no neurocutaneous stigmata.

Two typical events were captured on video electroencephalogram (EEG). While the patient was lying down, he woke, crying out, sat up, and rubbed his nose. This lasted for a few seconds and was followed by facial flushing, tachycardia, tachypnea, and then facial pallor. The patient was then seated and proceeded to have an altered level of consciousness where he responded inappropriately to questions and was not able to recall a memory word. The entire event lasted 40 to 60 seconds and ended with the patient confirming that the event was over. A postictal period was not observed.

The video EEG captured two stereotypical events with onset of symptoms associated with focal left temporal lobe spike waves followed by semirhythmic slowing over the left temporal lobe throughout the event (Figure 1). The patient touched his nose during the first few seconds of the recording; the period of unresponsiveness that followed coincided with a gradual spread of slowing over the majority of the left hemisphere. Postictal slowing was seen over the left temporal lobe. The patient was prescribed carbamazepine $200 \mathrm{mg}$ by mouth twice daily and has been seizure free for 1 year after initiation of medication. MRI of the brain was normal (Figure 2).

\section{DISCUSSION}

To our knowledge, this is the first description of a complex partial seizure that begins with an aura of pain at the tip of the nose followed by autonomic changes in the form of facial flushing, pallor, and tachycardia, and concludes with unresponsiveness. This is followed by an eventual return to baseline with no postictal phase. A literature review has revealed only one similar report, in which a patient presented with focal pain to the right ala of the nose with contralateral eye and head version followed by generalized tonic activity with no reported vasomotor or autonomic changes. ${ }^{1}$

The left temporal focus of the EEG does suggest a few different sources of the seizure onset and helps with localization given the normal MRI, although the ictal semiology does not help with lateralization of the ictal symptomatogenic zone. The initial nose pain is followed by prominent autonomic symptoms, particularly vasomotor changes. Autonomic symptoms, particularly ictal flushing, are reported as ictal phenomena of partial seizures in children and can localize to the temporal lobes with autonomic symptoms thought to result from seizure spread to the hypothalamus. ${ }^{3}$ It may be possible that the painful sensation is a result of a focal discharge from the amygdala, resulting in an experiential

From the Division of Pediatric Neurology, Department of Pediatrics (ES, DBS, HG); Division of Neurology, Department of Medicine (AA), Faculty of Medicine and Dentistry; Comprehensive Epilepsy Program (DBS), University of Alberta, Edmonton, $\mathrm{AB}$, Canada.

ES and AA were equally responsible for the work described in this paper. Received April 22, 2015. Final Revisions Submitted OCtober 28, 2015. Correspondence to: Helly Goez, Division of Pediatric Neurology, Department of Pediatrics, University of Alberta, 4-516 Edmonton Clinic Health Academy, Edmonton, AB, Canada T6G 1C9. Email: goez@ualberta.ca 

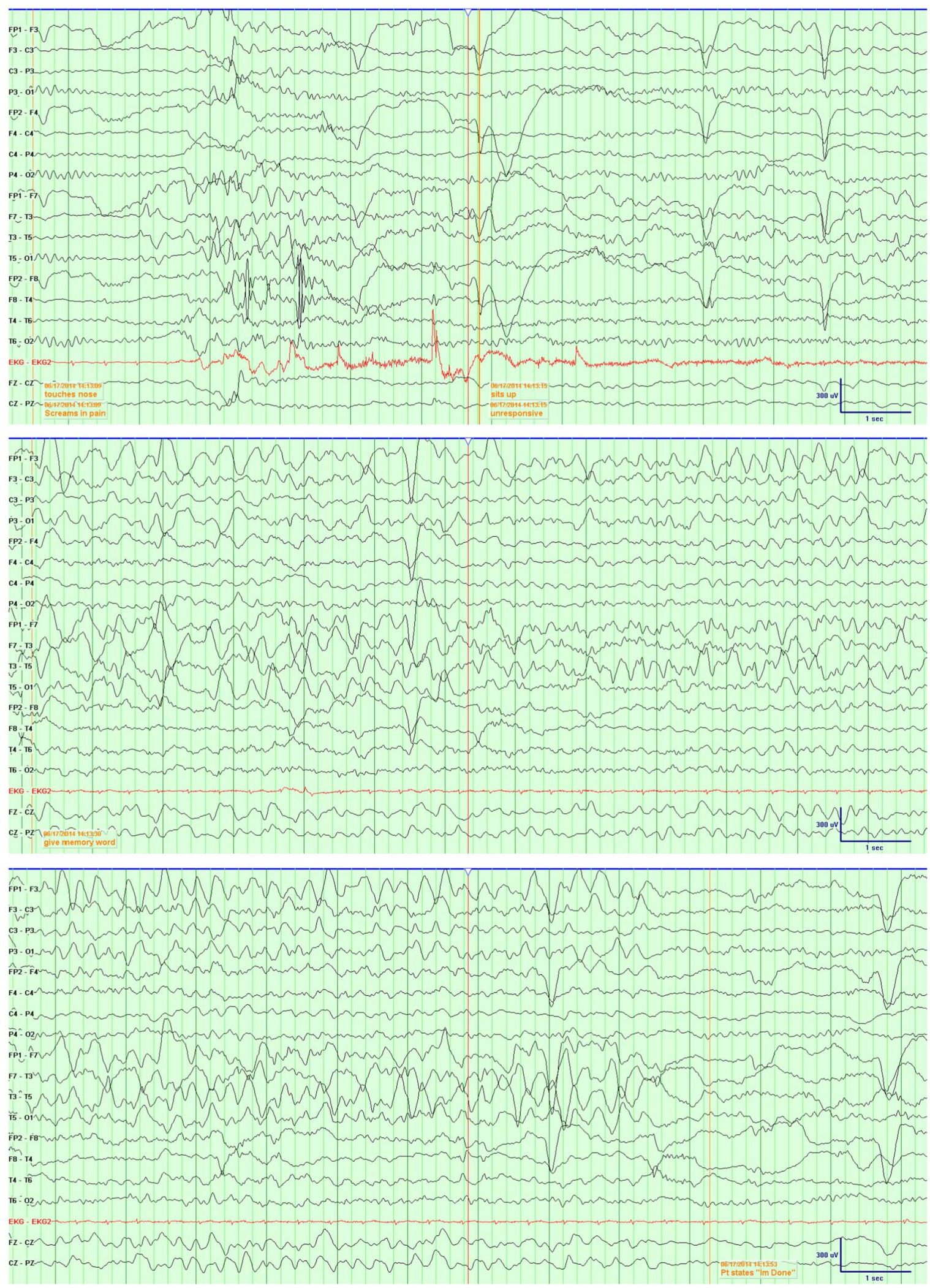

Figure 1: Horizontal bipolar montage. The beginning $(A)$, middle $(B)$, and end $(D)$ of the event are shown in the three panels. The event starts while the patient is asleep; he touches his nose, screams in pain, and sits up. This correlated with discharges particularly in the left temporal lobe. 


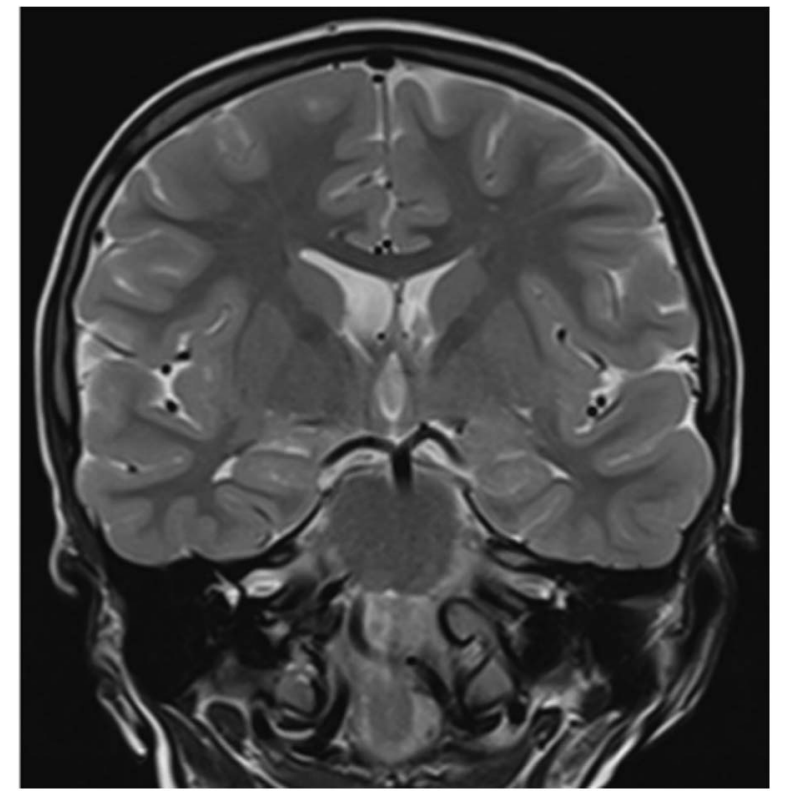

Figure 2: Coronal T2-weighted magnetic resonance imaging. A normal temporal lobe and insular cortex is seen with no evidence of a lesion.

phenomena or a feeling related to a past experience. Experiential seizures are reported to lateralize to the right amygdala. ${ }^{4}$ Painful somatosensory seizures have been shown to either originate or spread to the posterior insular cortex and/or the secondary somatosensory areas. ${ }^{5}$ From there, the seizure could spread to the amygdala or the hypothalamus thereby, resulting in experiential and autonomic phenomena. Finally, an ictal onset within the right temporal lobe with rapid spread to the left cannot be discounted either.

This case report raises a potentially interesting underlying connection between neuropathic pain and epilepsy. There is a striking similarity between our patient's presentation and that of the brief stereotyped pain with associated vasomotor changes seen in cranial neuralgias. Although an absence of unilateral pain and a precipitating trigger would make the diagnosis of cranial neuralgia unlikely, it should still be considered as a potential diagnosis. Furthermore, antiepileptic drugs can be used to treat seizures or neuropathic pain. The mechanism is thought to be through suppression of electrical activity of hyperexcitable neurons that may be seen in either situation. We would therefore suggest that, in a patient presenting with focal, stereotyped pain suspicious of neuralgia, particularly if dyscognitive features are present, further investigations in the form of an EEG and brain imaging should be considered.

\section{Disclosures}

ES, AA, DBS, and HG do have nothing to disclose.

\section{REFERENCES}

1. Young GB, Blume WT. Painful epileptic seizures. Brain. 1983;106:537-54.

2. Nair DR, Najm I, Bulacia J, Luders H. Painful auras in focal epilepsy. Neurology. 2001;57:700-2.

3. Fogarasi A, Janszky J, Tuxhorn I. Autonomic symptoms during childhood partial epileptic seizures. Epilepsia. 2006;47:584-8.

4. Gloor P. Experiential phenomenal of temporal lobe epilepsy. Facts and hypotheses. Brain. 1990;113:1672-94.

5. Mantovant A, Mauquiere F, Mazzola L, Garcia-Larrea L, Catenoix H, Ryvlin P, et al. On the origin of painful somatosensory seizures. Neurology. 2015;84:594-601. 